\title{
A Neutral Atom Moving in an External Magnetic Field Does Not Feel a Lorentz Force
}

\author{
Manfred Fähnle \\ Max Plank Institute for Intelligent Systems, Heisenbergstr, Stuttgart, Germany
}

Email address:

faehnle@is.mpg.de

\section{To cite this article:}

Manfred Fähnle. A Neutral Atom Moving in an External Magnetic Field Does Not Feel a Lorentz Force. American Journal of Modern Physics. Vol. 6, No. 6, 2017, pp. 153-155. doi: 10.11648/j.ajmp.20170606.17

Received: November 8, 2017; Accepted: November 28, 2017; Published: December 20, 2017

\begin{abstract}
A Lorentz force is generally exerted on a moving charged particle in an external magnetic field. But it is often asked whether also a neutral atom can feel a Lorentz force when moving straightly in an external magnetic field. It is shown that this is definitely not the case.
\end{abstract}

Keywords: Lorentz Force on Charged Particle, Lorentz Force on Neutral Atom, Expectation Values of Velocities

\section{Introduction}

It is well known $[1,2]$ that a magnetic field B exerts a Lorentz force on a moving particle with charge $\mathrm{q}$,

$$
\mathrm{F}_{\mathrm{L}}=\mathrm{q} / \mathrm{c}(\mathrm{v} \times \mathrm{B}) .
$$

Nevertheless, it is often asked whether a neutral atom can also feel a Lorentz force when moving in a magnetic field B. Even experts mean sometimes that these atoms feel a Lorentz force. The arguments are that a neutral atom consists of charged particles (nucleus and electrons) which all feel Lorentz forces, and that the effect of these Lorentz forces on the movement of the atom possibly do not cancel each other, because the mass of the nucleus is different from the mass of the electron, and because the expectation values of the positions of these particles appear at different positions. The Lorentz force is, e.g., discussed in the theory of magnetoelastic spin-phonon modes [3], in which the collective phonon-type movements of the atoms in a solid in the presence of a magnetic field B is investigated. The theory was originally formulated for the collective phonon-type movements of the charged ions of an ionic ferromagnet in an external magnetic field. In this context the appearance of Lorentz forces on the moving charged ions is clear. The theory was later applied [4] to itinerant ferromagnets like $\mathrm{Ni}$ or Fe. In these materials the valence electrons build atomic magnetic moments, and these atomic magnetic moments produce an internal magnetic field. The question then arises whether the atoms (which are neutral) feel a Lorentz force in this field. However, it was shown that one would have a double counting problem when including the Lorentz forces acting on the valence electrons in the magnetic field produced by themselves. Therefore only the Lorentz force acting on the unit with charge q consisting of the nuclear charge and the charges of the electrons in the closed electronic shells are considered [4]. The problem of double counting is known in the context of many-electron theory. There the electrostatic interaction of an electron with all electrons is described by the Hartee potential, which erroneously contains also the interaction energy of this electron with itself. With the so-called self-interaction correction $[5,6]$ it is attempted to correct this. However, thereby appears a series of complications. Without an exact self-interaction correction there are in some systems (e.g., 4f rare-earth elements and transition-metal oxides) artificial results, for instance, the favoring of an unrealistic delocalized state over a localized state. Therefore artificial effects most probably also would appear when the above discussed double counting would not be avoided.

Because in the theory of magnetoelastic spin-phonon modes the influence of the Lorentz force on the movement of the atoms is taken into account, these modes have well-defined angular momenta [3, 4]. In the usual theory of linearly-polarized phonons this effect is not taken into account, and therefore these phonons do not have well-defined angular momenta [7]. The question remains whether a neutral atom moving in an external field feels a Lorentz force, because there no double counting problem arises. In the present 
paper it is shown that this is definitely not the case. One will see that this proof is far from being trivial. This proof requires the quantum mechanical calculation which I will present in the next Section. There is no other type of proof, e.g., by a classical calculation.

\section{Theory}

To investigate the question raised in the Introduction one has to perform a quantum mechanical calculation, because an atom is a quantum mechanical particle. Thereby the quantum mechanical uncertainty relations have to be taken into account If this particle was in a state with well-defined momentum, i.e. well-defined velocity, then it would be described by a plane wave. Then the position of the atom would not be well-defined, the atom could be at all positions with equal probability. This would not correspond to the common notion of a particle. To construct a state which corresponds to such a notion one has to consider a wave packet by a superposition of plane waves with various wavevectors. Then the atom would be in a particle-like state, but neither the position nor the momentum, i.e., the velocity would be well-defined. Then it is natural to look at the expectation values for the position and for the velocity. If the expectation value of the velocity changes in time when the atom, i.e., the wavepacket moves in an external magnetic field, then the atom experiences a Lorentz force. It is natural to describe the motion of the wavepacket by looking at the centre of gravity of the moving neutral atom. The movement of this centre of gravity can be discussed by placing all masses there and by exerting on it the sum of all forces acting on the nucleus and on the various electrons of the neutral atom. Because all charged components of the atom feel Lorentz forces when the atom moves in an external magnetic field, we have to calculate the sum of the expectation values of the individual Lorentz forces. Thereby we use Ehrenfest's theorem [8,9] in the form that the expectation value of the position of a quantum mechanical particle obeys a classical law, i.e., it is given by Newton's equation of motion

$$
\mathrm{md}^{2}\langle\mathrm{r}\rangle / \mathrm{dt}^{2}=\mathrm{md}\langle\mathrm{v}\rangle / \mathrm{dt}=\langle\mathrm{F}\rangle .
$$

Here $\mathrm{m}$ is the mass of the considered particle (nucleus or electrons of the neutral atom), and $\mathrm{F}$ is the force acting on it. It is now assumed that the only forces acting on the various particles of the neutral atom are the Lorentz forces $F_{L}$, no other external forces. This gives

$$
\mathrm{md}^{2}\langle\mathrm{r}\rangle / \mathrm{dt}^{2}=\mathrm{md}\langle\mathrm{v}\rangle / \mathrm{dt}=\left\langle\mathrm{F}_{\mathrm{L}}\right\rangle=\mathrm{q} / \mathrm{c}(\langle\mathrm{v}\rangle \times \mathrm{B}) .
$$

This means that one has to calculate the expectation values of the velocities of the particles. Let us first consider a situation in which the centre of gravity of the system is at rest. In the electronic density functional theory the behavior of single electrons in single-electron states are discussed, which feel an effective Kohn-Sham potential [10]. The expectation value of the velocity then is defined as

$$
\langle\mathrm{v}\rangle=-\int \Psi^{*} \hbar \mathrm{i} \vec{\nabla} \Psi \mathrm{d}^{3} \mathrm{r},
$$

where $-i \hbar \vec{\nabla}$ is the quantum mechanical velocity operator.

In the literature these expectation values are sometimes calculated explicitly, by using the symmetry properties of the system. For instance, in a spherically symmetric situation like in an atom without external field it can be shown that $\langle\mathbf{v}\rangle$ is zero. The question is how to proceed in situations which are not spherically symmetric. For, instance, an external magnetic field breaks the spherical symmetry of an atom [11], or in a molecule there is no spherical symmetry [12]. However, from a physical point of view it is clear that $\langle\mathbf{v}\rangle$ is zero for a stationary bound state of an electron in a situation in which the centre of gravity of the whole system is at rest.

For $\langle v\rangle$ not equal to zero the electron would leave the system, i.e., it would not be in a stationary bound state. Altogether this means that in the situation where the centre of mass of the system does not move, there is not resulting expectation value of the Lorentz force, because all expectation values of the velocities of the involved particles (electrons and nucleus) are zero.

Let us now consider the situation in which the centre of gravity moves straightly with constant velocity $\mathrm{v}_{\mathrm{c}}$, or - more accurately written, see above - with constant velocity $\left\langle\mathrm{v}_{\mathrm{c}}\right\rangle$. Then the expectation values of all involved particles are $\langle v\rangle=v_{c}$. Nevertheless, the expectation value of the sum of all Lorentz forces acting on the various particles is still zero. The reason is that the nucleus has a charge $\mathrm{q}$, and the sum of the charges of all the electrons of the neutral atom is -q, so that $\left\langle\mathrm{F}_{\mathrm{L}}\right\rangle$ is indeed zero although the centre of gravity is moving.

The particles of the atom feel also the Coulomb interactions $f_{i j}=-f_{j i}$ between them. They occur also in the sum of forces acting on the centre of gravity. However, to calculate their total contribution to the force acting on the centre one has to sum over all $\mathrm{i}$ and $\mathrm{j}$, and this gives zero.

Altogether this means that the centre of gravity of a moving neutral atom does not feel an effective Lorentz force in an external magnetic field, although the mass of the nucleus is different from the mass of the electrons and although the expectation values of the positions of the various particles are at different positions (see Introduction).

\section{Conclusion}

It has been shown that neutral atoms moving in an external magnetic field do not experience a resulting Lorentz force acting on their centre of gravity. Therefore the movement of a neutral atom with constant velocity relative to an external magnetic field at rest is not affected by the magnetic field. The story is different from the one of moving neutral atoms in an internal magnetic field which is produced by the valence electrons of the atoms. As shown in the Introduction, thereby the Lorentz forces on the valence electrons which build the atomic magnetic moments should not be taken into account when calculating the sum of Lorentz forces acting on the centre of gravity of the system, because otherwise one would run in a double counting problem. Because of this there remains indeed a resulting expectation value of the Lorentz forces acting on the centre of gravity, which is described by the Lorentz force acting on a unit with charge $\mathrm{q}$ which consists 
of the nuclear charge and of the charges of the electrons in the closed electronic shells.

\section{Acknowledgements}

The author is indebted to Lifa Zhang for many discussions.

\section{References}

[1] L. D. J. Griffiths, Introduction to electrodynamics, 3rd ed. (Pearson/Benjamin Cummings, San Francisco, 2008) 'International Edition'-Cover.

[2] J. D. Jackson, Classical Electrodynamics, 3rd ed., New York, 1989.

[3] L. Zhang and Q. Niu, Angular momentum of phonons and the Einstein-de Haas effect, Phys. Rev. Letters 112, 085503 085507 (2014).

[4] T. Tsatsoulis, C. Illg, M. Haag, B. Y. Mueller, L. Zhang, and M. Fähnle, Ultrafast demagnetization after femtosecond laser pulses: Transfer of angular momentum from the electron system to magnetoelastic spin-phonon modes, Phys. Rev. B 93, 134411-134417 (2016).

[5] A. Svane and O. Gunnarson, Transition-metal oxides in the self-interaction corrected density-functional theory, Phys. Rev. Letters 65, 1148 - 1151 (1990).

[6] W. M. Temmermann, Z. Szotek and H. Winter, Band-structure method for 4 f electrons in elemental Pr metal, Phys. Rev. B 47, 1184 - 1189 (1993).

[7] A. McLellan, The quantum theory of crystal deformations: The harmonic approximation and rotational invariance, J. Phys. C: Solid State Phys. 21, 1177 (1988).

[8] P. Ehrenfest, Bemerkung über die angenäherte Gültigkeit der klassischen Mechanik innerhalb der Quantenmechanik, Zeitschrift für Physik 45, 455 - 457 (1927).

[9] A. Messiah, Quantum Mechanics, Elsevier, North Holland, Amsterdam, 1962.

[10] W. Kohn and L. J. Sham, Self-Consistent Equations including Exchange and Correlation Effects, Phys. Rev. 140, A1133 A1138 (1965).

[11] H. Lehmann and N. H. March, The hydrogen atom in intense magnetic field: Excitons in one and three dimensions, Pure \& Applied Chem. 57, 457 - 462 (1995).

[12] J. E. Rosenthal and G. M. Murphy, Group Theory and the Vibrations of Polyatomic Molecules, Rev. Mod. Phys. 8, 317 346 (1936). 